\title{
The central mechanism of tadalafil vs Chaihu- Shugan-San capsule for psychogenic erectile dysfunction: a magnetic resonance imaging study protocol of a randomized placebo-controlled trial
}

\section{Feiqiang Ren}

Chengdu University of Traditional Chinese Medicine

\section{Ziyang Ma}

Chengdu University of Traditional Chinese Medicine

\section{Ling Zhang}

Chengdu University of Traditional Chinese Medicine

\section{Liang Dong}

The School of Medical and Life Sciences of Chengdu University of Traditional Chinese Medicine

\section{Diang Chen}

Hospital of Chengdu University of Traditional Chinese Medicine

Degui Chang ( $\sim$ changdegui123@163.com )

Chengdu University of Traditional Chinese Medicine

\section{Peihai Zhang}

Hospital of Chengdu University of Traditional Chinese Medicine

\section{Guangsen Li}

Hospital of Chengdu University of Traditional Chinese Medicine

Xiaopeng Huang

Hospital of Chengdu University of Traditional Chinese Medicine

\section{Yaodong You}

Hospital of Chengdu University of Traditional Chinese Medicine

\section{Xunjun Yu}

The School of Medical and Life Sciences of Chengdu University of Traditional Chinese Medicine

\section{Fang Yang}

The School of Medical and Life Sciences of Chengdu University of Traditional Chinese Medicine Zhengjie Li

The Tuina School of Chengdu University of Traditional Chinese Medicine

\section{Method Article}


Keywords: Erectile dysfunction, psychogenic ED, MRI, Randomized controlled trial

Posted Date: January 23rd, 2019

DOI: https://doi.org/10.21203/rs.2.241/v1

License: (c) (1) This work is licensed under a Creative Commons Attribution 4.0 International License. Read Full License 


\section{Abstract}

Background: Erectile dysfunction (ED) affects many adult men worldwide. Many studies on the brain of psychogenic ED have shown significant cerebral functional changes and reduced volume of gray matter and white matter microstructural alterations in widespread brain regions. We design this study to provide both an enhanced level of evidence of central mechanism research and an evidence-based treatment option for patients suffering from psychogenic ED. Furthermore, we also aim to provide a new evidence as well as an innovation of the clinical treatment in psychogenic ED.

Methods: This study is designed as a multi-center, three-arms, randomized trial. From the perspective of psychogenic ED, we will divide patients into three groups, which are placebo group, tadalafil group and CHSGS group. 135 patients will be randomly allocated to receive placebo, CHSGS capsule or tadalafil oral pharmacotherapy. After the period of 4-week treatment, the outcome of primary assessment changes in the IIEF-5, EHS and QEQ total scores from baseline. Secondary assessments include the SEAR, HAMA-14, HAMD-17 scores, response rate of the patients and their partners. Apart from brain MRI from baseline, we will also attach great importance to other changes in the follow-up four weeks.

Discussion: We expect that the results of this trial can provide both an enhanced level of evidence for central mechanism research and an evidence-based treatment option for patients suffering from ED, as well as to provide an innovation of the clinical treatment in psychogenic ED.

Trial Registration: chictr.org.cn, ChiCTR-IOR-1800018301. Registered on 10 September 2018.

Keywords: Erectile dysfunction, psychogenic ED, MRI, Randomized controlled trial.

\section{Background}

Defined as the current or consistent presence of the disability to attain or/and maintain a penile erection sufficient for sexual satisfaction[1,2] or satisfactory sexual performance which lasts for at least 6 months in accordance with the Diagnostic and Statistical Manual of Mental Disorders, Fifth Edition (DSM-5), erectile dysfunction (ED) or impotence is one of the most common male sexual dysfunctions. The prevalence of ED is increasing at an alarming rate in all age groups over the world, with a worldwide incidence of $12 \%$ of men under the age of $59,22 \%$ of men between 60 and 69 years of age and $30 \%$ of men over 69 years old[3]. A conservative research reported that 322 million individuals will be affected by ED around the world by the year of 2025[3]. ED has been a great social problem and an important health care issue because of its huge impact upon the quality of life (QOL)[4], overall psychological well-being of both sexual activity participants and its association with multiple cardiovascular diseases and atherosclerosis[3-8].

According to the current guidelines, tadalafil [12-16] is the first-line oral pharmacotherapy for ED. It could take action from various species in vivo and in vitro by increasing the intracellular concentrations of cGMP by amplifying the endogenous NO-cGMP pathway $[9,17]$. The cell molecular mechanisms involved 
have been reviewed in detail elsewhere[18]. The fact that CNS mechanisms play an important role for erection and penile as drugs targets for ED has been accepted[11, 13, 18]. There are many studies on the brain MRI of psychogenic ED[7, 19-31].

Otherwise, Chinese herbs have been widely accepted for management of impotence in China for thousands of years and have been gradually recognized as a complementary therapy. Clinical effects has demonstrated that Chaihu-Shugan-San (CHSGS) capsule treatment can improve the symptoms and QOL of patients with psychogenic ED. Animal studies have confirmed that CHSGS is an antidepressant[32]. Traditional Chinese medicine believes that CHSGS could regulated the mental disorders. Despite significant progress, the mechanism aiming at CNS targets treated by tadalafil or CHSGS capsule is still unknown.

Normal erectile function is a result of the harmonious regulation and control of the central nervous system (CNS), which includes the supraspinal centres, the spinal cord and peripheral nerves. In the last two decades, exploring the role of CNS in sexual arousal by functional and structural neuro-imaging studies, the magnetic resonance imaging (MRI) [20] such as diffusion tensor imaging (DTI)[21, 23-25], functional MRI (fMRI) and resting-state fMRI attracted many researchers[23-25]. Since 2002, compared with healthy subjects (HS), much researchers have found that psychological ED patients showed significant changes in cerebral functions. What is more, reduced volume of gray matter and white matter microstructural alterations in widespread brain regions mediating controlled and regulated the processes of erection have also been found in many regions of the human brain of the psychogenic ED[21-28, 33]. MRI techniques are widely used to study the central mechanism and show positive result with premature ejaculation[34], rheumatoid arthritis[35], functional dyspepsia[36], fibromyalgia[37], addictive disorders[38] and especially in depressive patients treated with paroxetine[39]. Therefore, we hypothesized that compared with the tadalafil or CHSGS or placebo alone, to treat psychogenic ED with the CHSGS or tadalafil will be more effective due to its specific brain function modulation.

\section{Methods}

\section{Study design}

This is a multi-center, three-arms, randomized trial which was designed following the Consolidated Standards of Reporting Trials (CONSORT) Statement recommendations and the Standardized Protocol Items: Recommendations for Interventional Trials (SPIRIT) guidelines[40-42].

This study will be carried out in the Hospital of Chengdu University of Traditional Chinese Medicine and Sichuan Integrative Medicine Hospital. During the 4-week treatment, patients in three interventions will receive a continuous oral medication with tadalafil, or CHSGS or placebo. Both the MRI scan and outcome assessment will be fulfilled at two time points, including the baseline and the end of the intervention treatments (Figs. 1 and 2). This trial is reported in accordance with the Standard Protocol Items: Recommendations for Intervention Trials (SPIRIT) guidelines [40, 42]. All of the participants will be asked to sign an informed consent before the trial that will contain an introduction of pED and ED, the 
inclusion and exclusion criteria, and a detailed introduction to the interventions. Furthermore, participants have the right to desert the trial at any time. Ethical approval has been obtained from the Institutional Review Board (IRB) of the Hospital of Chengdu University of Traditional Chinese Medicine (Approval No. 2018KL-064) and the Sichuan Integrative Medicine Hospital (Approval No. 2017KY-02) conducted in accordance with the Helsinki Declaration, and it has been registered under the identifier No.ChiCTR-IOR1800018301 on the Chinese Clinical Trial Registry on September 10, 2018.

\section{Sample size}

Due to the lack of reference on the expected effect size of using CHSGS to treat erectile dysfunction evaluated in this study, we did not estimate the sample size based on a power calculation. According to previous studies, International Index of Erectile Function 5 (IIEF-5) scores of ED patients increased 6.0-7.1 after tadalafil $[13,15]$. Therefore, we predicted ascension of IIEF- 5 score by 6.5 points after oral tadalafil or CHSGS capsule, a reduction by 2.5 points after placebo. According to the calculation with PASS software (Version 11.0, NCSS, LLC. Kaysville, UT, USA) in a 1:1:1 ratio, when $\alpha=0.05,1-\beta=0.8$ with the standard deviation to be 3.8 , the effect size is 0.2550 of 41 participants in each group. Considering a dropout rate of $10 \%$ and according to our previous study, a sample size of 135 patients at baseline will be planned in this study.

According to the sample size of previous BOLD-fMRI[23], TDI[21], and fMRI studies, 12-15 subjects is the very reasonable sample size with stable statistical effect[29,30]. Considering the dropout rate and loss of data as to head motion during MRI scans, 15 participants from each group will undergo MRI scans in this study.

\section{Randomization}

Qualified participants will be randomly assigned to either the tadalafil group, the placebo group or the CHSGS capsule group in a ratio of 1:1:1. Random numbers will be generated by a random number generator in the SPSS statistical software package (Version 22.0, SAS Institute Inc.), which will be operated by a third party who is uninvolved with the treatment and data collection. The drawn letters (A or $B$ or $C$ ) will be placed into opaque envelopes labeled with sequential numbers. The envelopes will be sealed and remain in numerical order in a safe place till the completion of this study. The same researcher (not involved in the study) will prepare the envelopes.

\section{Blinding}

The medicine will be specially handled and labeled by the Pharmacy Department in the Hospital of Chengdu University of TCM to guarantee that the patients and the practitioners included in the study will maintain completely blinded as to the identity of the treatment administered. Besides, the all practitioner will be prohibited to communicate with participants about the information of this trail.

\section{Participant selection}




\section{Recruitment requirement}

All psychogenic ED patients are recruited at the outpatient department in the Hospital of Chengdu University of Traditional Chinese Medicine (TCM) and Sichuan Integrative Medicine Hospital via advertisements in newspapers and via the Internet from November 2018 to January 2019.

All patients must be in a stable heterosexual relationship for at least 1 year, and must be estimated by as follows: (i) a detailed history asking, including psychosocial history (paying attention to the patient's expectation of his own sexual performance and his general attitude and knowledge about sex, etc.) and interviewing the patient's partner, medical history, mainly about relevant drug history (including tobacco, alcohol, or illicit drug use and so on), and surgical disorder; (ii) a careful and detailed physical examination, especially the urology and andrology examination and rectify any misconceptions the patient might have about the relationship between penile length, masculinity, and erection; (iii) a basic laboratory test, containing a nocturnal penile tumescence (NPT) test, a penile duplex Doppler ultrasonography (DUS) with PSV measurements before and after ICl of prostaglandin, as well as routine blood examinations, thyroid-stimulating hormone, serum prostatic specific antigen (PSA), and the serum sexual hormone level (sexual hormone binding globulin, free or/and total testosterone, follicle-stimulating hormone, luteinizing hormone, estrogen, and prolactin); (iv) a psychophysical condition evaluated by 2 separate experienced psychologists, and (v) a series of questionnaires, mainly concerning about the International Index of Erectile Function 5 (IIEF-5), a self-esteem and relationship questionnaire (SEAR), the erection hardness score (EHS), the erection quality questionnaires (QEQ), the 14-item Hamilton Rating

Scale for Anxiety (HAMA-14) and the 17-item Hamilton Rating Scale for Depression (HAMD-17). All of the above must be by checked before being enrolled in this study.

\section{Inclusion criteria}

Patients must meet the following diagnostic criteria from the current guidelines, including: (i)men be right-handed and the age ranged from 20 to 40, (ii) men be diagnosed as psychogenic ED (DSM-5), (iii) men must be in a stable heterosexual relationship for at least 1 year with his regular sexual partner, (iv) penile erection can occur in the circumstance of masturbation or audiovisual stimulation, (v) men who meet the TCM diagnosis of stagnation of liver-qi", (vi) men who agree to complete all demand during the study period, (vii) sign an informed consent form.

\section{Exclusion criteria}

Exclusion criteria for the forthcoming study are as follows: (i) be diagnosed as organic ED rather than psychogenic disorder, (ii) a current diagnosis or history of drug or alcohol dependence; (iii) use of any medication that might impact sexual function during the previous 30 days before enrolled in this study; (iv) any physical illness as assessed by personal history and laboratory analysis finding any history of serious psychiatric, neurological, cardiovascular, respiratory, gastrointestinal or renal diseases or hepatic disorders or significant physical disorders; (v) participated in any other current clinical trials; (vi) had any contraindications for MRI scan. 


\section{Intervention and comparison}

\section{Tadalafil group}

Patients will be provided a once-daily treatment with $5 \mathrm{mg}$ tadalafil added to the capsule until 4 weeks.

According to the current guidelines, the therapeutic interventions for ED are basic treatment, drug treatment, physical treatment and surgical treatment[12]. Drugs are phosphodiesterase type 5 inhibitors such as tadalafil, sildenafil, vardenafil, avanafil, which have been regarded as the first-line oral pharmacotherapy for $E D[17,18]$. Some previous clinical research have confirmed that patients and partners prefer tadalafil to sildenafil in the treatment of ED[11, 13-16, 43, 44].Therefore, we selected tadalafil as the positive control group as well as the treatment group. Smashed tadalafil will be mixed with starch powder and stir well into a container in order to be made into capsule.

\section{CHSGS group}

Patients will be provided three-daily treatment with $5 \mathrm{~g}$ CHSGS added to the 5 capsule each time until 4 weeks.

The CHSGS preparation contained the gathering of the following components: $6 \mathrm{~g}$ Bupleurum Chinese root, 4.5g Rhizoma Chuanxiong, 4.5g Fructus Aurantii, $6 \mathrm{~g}$ Pericarpium CitriReticulatae, $4.5 \mathrm{~g}$ Paeonia, $1.5 \mathrm{~g}$ Glycyrrhizaeuralensis root and $5 \mathrm{~g}$ Cyperusrotundus. All CHSGS herbal components were obtained from the Hospital of Chengdu University of TCM. The herbal components were identified by an expert in order to fulfill the quality requirements of the Pharmacopoeia of the People's Republic of China (2015 edition). CHSGS and its components were individually decocted in boiling water for $30 \mathrm{~min}$, concentrated and vacuum-dried to form a paste, and were subsequently combined into a paste containing $8 \mathrm{~g}$ crude extracts per gram. Starch powder was mixed into a container. Stir it well and make it into capsule.

\section{Placebo group}

Patients will be provided three-daily treatment with the $5 \mathrm{~g}$ mixed starch powder added to the 5 capsule each time until 4 weeks.

It is known that placebos are the best comparison between clinical controlled trials. Therefore, we adopted this placebo and standardized its performance. The placebo components were obtained from the Hospital of Chengdu University of TCM. The standard operating procedures (SOP) are listed as follows: (i) the materials are starch, (ii) add the liquid base: place the mixed starch powder into a container, add honey and stir well to make it into capsule. The above materials made shared the same character with the CHSGS capsule in terms of appearance, weight and taste.

\section{Outcome assessment}

The following outcomes will be assessed by independent assessors, who had been trained before participating in this study and blinded to the randomization. All outcome data for participants whether 
completed or withdrawn during the study will be collected and recorded in the case report form (CRF).

\section{Symptom assessment}

Based on various clinical studies, the primary symptoms of the ED patients will be assessed on mean changes from baseline to end of observation in the International Index of Erectile Function 5 (IIEF-5) total scores $[45,46]$, which has been widely used and recommended as a primary outcome for clinical trials of ED. Furthermore, the quality of erection questionnaire (QEQ) [47-49] and the erection hardness score (EHS) total scores will also be included in this study[47, 50]. In terms of the assessment of symptom severity of erectile function for all the participants in this study, the secondary outcome will be based on the selfesteem and relationship (SEAR) questionnaire[51]. As to the level of anxiety and depression of all the participants in this study, it will be evaluated by two independent psychologists within the questionnaires of the 14-item Hamilton Anxiety Rating Scale (HAMA-14) and the 17-item Hamilton Depression Rating Scale (HAMD-17) [52, 53].

\section{MRI scan}

The MRI scanning of the brain will be executed on a 3.0-T Siemens MRI scanner (General Electric Company, America) at the Hospital of Chengdu University of Traditional Chinese Medicine, Chengdu, China, including the magnetic resonance (MR) diffusion tensor imaging (DTI), T1-weighted, a restingstate blood oxygenation level dependent MRI (BOLD-MRI) data of all participants in this study.

T1-weighted structure images in 3D Magnetization-Prepared Rapid-Gradient-Echo (MPRAGE) Images will be firstly collected before resting-state scanning with the following sequence parameters: field of view $(F O V)=240 \otimes 240 \mathrm{~mm}^{2}$, repetition time $(T R)=1900 \mathrm{~ms}$, echo time $(T E)=2.26 \mathrm{~ms}$, and matrix $=256 \varangle 256$.

DTI will be applied along 63 non-linear directions ( $b$ value $=1000 \mathrm{~s} / \mathrm{mm} 2$ ) together with an acquisition without diffusion weighting ( $b$ value $=0 \mathrm{~s} / \mathrm{mm}^{2}$ ), and the scanning parameters are as follows: FOV= $230 \times 230 \mathrm{~mm}^{2}, \mathrm{TR}=8900 \mathrm{~ms}, \mathrm{TE}=84 \mathrm{~ms}$, and matrix $=256 \times 256$.

The resting-state BOLD-MRI will be obtained by using Gradient-Recalled Echo-Planer Imaging (GER-EPI) with the following sequence parameters: $T R=2000 \mathrm{~ms}, T E=30 \mathrm{~ms}$, inverse angler $(I A)=90^{\circ}$, number of slice $=32$, slice thickness $(\mathrm{ST})=5 \mathrm{~mm}, \mathrm{FOV}=240 \rrbracket 240 \mathrm{~mm}^{2}$, matrix $=64 \llbracket 64$, and total volumes $=400$.

\section{Safety assessment}

Adverse events (AEs) related to tadalafil treatment such as myalgia, back pain and flushing will be appropriately estimated and recorded by the observers during the trial. AEs will be managed by a specialized practitioner (do not participate in clinical data analysis) within 24 hours. The principal researcher (PH. Zhang) will make the final decision to terminate the trial if severe AEs occurs. All data for participants during the study and details of related and unexpected AEs (the time of occurrence, severity of $A E$, and suspected causes) will be collected and recorded in the case report form (CRF). Measures may 
vary from symptomatic treatment to case submission to the Research Ethics Committee within 48h depending on the severity of AEs.

\section{Quality control and Data collection}

Due to the fact that any nonstandard or bias input of clinical data can dominate the bias of results, two practitioners (to estimate the effect of treatment and the data authenticity) will independently gather the data with case report forms and original cerebrum MRI. The collected data will be input into a dedicated computer. The above process will maximize the reliability and safety of the all data. In order to guarantee the quality of the study, all practitioners will be required to have an official license for at least two years of protocol study and clinical experience.

\section{Data analysis}

\section{Clinical variables}

The clinical data will be analyzed with the help of SPSS22.0 (SPSS Inc., Chicago, IL, USA) by two blinded evaluators and the data analysis will be based on the intention-to-treat (ITT) principle regarding baseline characteristics. All the clinical data in this study will be presented as follows: (i) a continuous measurement data being presented as the mean \pm standard deviation (SD), the mean, SD, median and interquartile range. (ii) a categorical data being presented as case and percentages. Analysis tools include: the Cochran-Mantel-Haenszel (CMH) test or nonparametric test, an independent samples t-test, and a two-sided test. Also, analysis of covariance, covariance analysis or generalized estimating equations, $\chi 2$ and Linear regression will be applied on all available and suitable data ( $p$ value $<0.05$ is considered to be statistically significant).

\section{MRI data}

Based on our previous research's methods and acquisition of the brain MRI data, MRI data will be firstly preprocessed SPM12 software (SPM12, London, UK; http://www.fil.ion.ucl.ac.uk/spm/) performing on MATLAB 8.6 (Math works, Inc., Natick, MA, USA),Brain Voyager QXsoftware (Brain Innovation, The Netherlands)[54]. And then, for accurately investigate the different cerebral responses, including regional homogeneity (ReHo)[55], tract-based spatial statistics (TBSS)[56, 57], amplitude of low-frequency fluctuation (ALFF) [58], and functional connectivity (FC) will be used in this study. Finally, correlation analysis will be processed between clinical data and MRI image data.

Fig3 illustrates the data access and the analysis of the brain MRI data as well as the analysis methods of the primary and secondary outcomes.

\section{Discussion}

In this protocol study, the qualified participants are asked to maintain their current living habits and sexual performances, especially their diet, exercise and the quality of sleep. Meanwhile, they are required 
to fill out relevant questionnaires throughout the full observational process. To satisfy the requirements of the Rule of Ethics and reduce the bias caused by performance, the rest of the all clinical procedures maintain the same.

Seen from the current records and recent RCT results, tadalafil has an enduring effect and was the bestmarched choice between patients and their partner for treating psychogenic ED. However, its central mechanism remains unclear. Considering the importance of the brain for the psychogenic ED, we have designed the randomized controlled MRI trial to reveal the central mechanism of the psychogenic ED treated by tadalafil. In this study, we will compare the changes in brain function after administration of two interventions: the tadalafil and the CHSGS capsule.

Many regions of the human brain have been found to play a very important role in human sexual arousal as well as penile erection, emotion, and cognition. Over the last 15 years, MRI science has been widely used to disclose the cerebral mechanism of human sexual arousal especially the penile erection and investigate the brain function alteration in psychogenic ED and healthy subjects. Erection is known to be one of the most convincing and mischievous signs of the sexual response cycle. Relatively, ED not only can be conceptualized as an impairment in the arousal phase of sexual response[59], but also can be seen as a central and spinal center reflex, which can be sponsored by recruitment of penile afferents, both somatic and autonomic, and the changes of visual, imaginary stimuli, and olfactory. Several central transmitters[60] play a crucial role in erectile control and the main regions that control of male sexual arousal are summarized in Fig 4. Previous DTI studies showed that psychogenic ED had decreased or increased white matter microstructures in multiple brain regions[21]. Besides, brain networks neuroimaging studies also had showed that psychogenic ED had increased the small-worldness and modules in the left prefrontal cortex and limbic system including the right superior parietal gyrus, and the superior parietal gyrus[23-25].These brain networks were absolutely believed to be closely related to the brain sexual arousal of the psychogenic ED[60-63].

In order to guarantee the reliability of the result of this study, we adopt the quality key point control as follows: (i) patient in this study restricts the subjects to age between 20 to 40 and being right-handed for baseline homogeneity; (ii) sample size to obtain stable statistical power; 135 patients will be included in each group for clinical evaluation and 15 patients will be included in each group for the central mechanism study and receive MRI scans; and (iii) MRI scans; each scan will be performed in the afternoon with the same scanner and operator. During the scanning period, all participants will be asked to remain relaxed, keep their eyes closed and wear a birdcage head coil filled with sponge material and to stay still to reduce the effects of head movement. The scanning room maintains the noise less than 150 $\mathrm{dB}$ and the temperature between 18 and $22^{\circ} \mathrm{C}$, with humidity higher than $60 \%$.

In conclusion, this study is conducted in the purpose of supporting the concept of brain MRI alteration in psychogenic ED with tadalafil or CHSGS capsule. We expect that the results of this trial can provide both an evidence-based treatment option for patients suffering from ED, an enhanced level of evidence on 
which central mechanism research and to provide an innovation of the clinical treatment in psychogenic ED.

\section{Abbreviations}

ED: erectile dysfunction; EHS: Erection hardness score; QEQ: Quality of erection questionnaire; NPT: Nocturnal penile tumescence; SEAR: Self-esteem and relationship questionnaire; IIEF-5: International index of erectile function 5; penile DUS: penile Duplex doppler ultrasonography; HAMA-14: 14-item Hamilton Rating Scale for Anxiety; HAMD-17: 17-item Hamilton Rating Scale for Depression; AEs: Adverse events; MRI: Magnetic resonance imaging; DTI: Diffusion tensor imaging; BOLDMRI: Blood oxygenation level dependent MRI; fMRI: functional MRI; DOE: Degree of expectation; DOS: Degree of satisfaction; $\mathrm{CMH}$ : Cochran-Mantel-Haenszel test; fALFF: fractional Amplitude of Low-Frequency Fluctuation Analysis; TBSS: tract-based spatial statistics; QOL: Quality of life; RCT: Randomized clinical trial; ReHo: Regional homogeneity; SPIRIT: Standard Protocol Items; CHSGS: Chaihu-Shugan-San.

\section{Declarations}

\section{Ethics approval and consent for participation}

This study protocol has been approved by the Institutional Review Board of the Hospital of Chengdu University of TCM (Approval No. 2018KL-064), the Sichuan Integrative Medicine Hospital (Approval No. 2017KY-02) and has been registered under the identifier No.ChiCTR-IOR-1800018301 on the Chinese Clinical Trial Registry. Any modifications to the research protocol will be notified to this Human Research Ethics Committee. Informed consent will be obtained from each participant prior to enrollment. The study bases on the principles of the Declaration of Helsinki and Good Clinical Practice guidelines.

\section{Trial status}

Participant recruitment is currently ongoing. 3 participants have been recruited. Its deadline would correspondingly be postponed to February 2019.

\section{Consent for publication}

Not applicable.

\section{Availability of data and materials}

Not applicable. 


\section{Competing interests}

The authors declare that they have no competing interests.

\section{Funding}

The trial is financially supported by the National Natural Science Foundation of China (NO. 81774137). The role of the funding in the design is to collect and analysis the data.

\section{Authors' contributions}

$F R, Z M, P Z, D C$, and ZL have put forward the conception and design of the Trial and planned for the analysis of the data. $F R, P Z$, and $D C$ agreed in drafting this manuscript. $F R, Z M, L Z, X Y, Y Y, D C, X H, G L, L D$ and FY standing in the data Collection, TY and ZL standing in the data analysis, and were in the charge of recruitment and treatment of patients. Authors discussed, read, revised the manuscript, and approved the final manuscript.

\section{Acknowledgements}

None.

\section{References}

1.Glina S, Cohen DJ, Vieira M: Diagnosis of erectile dysfunction. Current opinion in psychiatry 2014, 27(6):394-399.

2.Rajkumar RP: The impact of disrupted childhood attachment on the presentation of psychogenic erectile dysfunction: an exploratory study. J Sex Med 2015, 12(3):798-803.

3.Bacon CG, Mittleman MA, Kawachi I, Giovannucci E, Glasser DB, Rimm EB: Sexual function in men older than 50 years of age: results from the health professionals follow-up study. Ann Intern Med 2003, 139(3):161-168.

4.Capogrosso P, Montorsi F, Salonia A: Erectile dysfunction in young patients is a proxy of overall men's health status. Current opinion in urology 2016, 26(2):140-145. 
5.Zusman RM: Cardiovascular data on sildenafil citrate: introduction. The American journal of cardiology 1999, 83(5a):1c-2c.

6.Feldman HA, Goldstein I, Hatzichristou DG, Krane RJ, McKinlay JB: Impotence and Its Medical and Psychosocial Correlates: Results of the Massachusetts Male Aging Study. The Journal of Urology 1994, 151(1):54-61.

7.Vicenzini E, Altieri M, Michetti PM, Ricciardi MC, Ciccariello M, Shahabadi H, Puccinelli F, Lenzi GL, Di Piero V: Cerebral vasomotor reactivity is reduced in patients with erectile dysfunction. Eur Neurol 2008, 60(2):85-88.

8.Shamloul R, Ghanem H: Erectile dysfunction. Lancet 2013, 381(9861):153-165.

9.Yuan J, Zhang R, Yang Z, Lee J, Liu Y, Tian J, Qin X, Ren Z, Ding H, Chen Q et al: Comparative effectiveness and safety of oral phosphodiesterase type 5 inhibitors for erectile dysfunction: a systematic review and network meta-analysis. Eur Urol 2013, 63(5):902-912.

10.Kim SC, Lee YS, Seo KK, Jung GW, Kim TH: Reasons and predictive factors for discontinuation of PDE5 inhibitors despite successful intercourse in erectile dysfunction patients. Int J Impot Res 2014, 26(3):8793.

11.Gong B, Ma M, Xie W, Yang X, Huang Y, Sun T, Luo Y, Huang J: Direct comparison of tadalafil with sildenafil for the treatment of erectile dysfunction: a systematic review and meta-analysis. Int Urol Nephrol 2017, 49(10):1731-1740.

12.Hatzimouratidis K, Amar E, Eardley I, Giuliano F, Hatzichristou D, Montorsi F, Vardi Y, Wespes E, European Association of U: Guidelines on male sexual dysfunction: erectile dysfunction and premature ejaculation. Eur Urol 2010, 57(5):804-814.

13. Hatzichristou D, d'Anzeo G, Porst H, Buvat J, Henneges C, Rossi A, Hamidi K, Buttner H: Tadalafil 5 mg once daily for the treatment of erectile dysfunction during a 6-month observational study (EDATE): impact of patient characteristics and comorbidities. BMC Urol 2015, 15:111.

14.Bai WJ, Li HJ, Dai YT, He XY, Huang YR, Liu JH, Sorsaburu S, Ji C, Jin JJ, Wang XF: An open-label, multicenter, randomized, crossover study comparing sildenafil citrate and tadalafil for treating erectile dysfunction in Chinese men naive to phosphodiesterase 5 inhibitor therapy. Asian J Androl 2015, 17(1):61-67.

15.Brock G, Ni X, Oelke M, Mulhall J, Rosenberg M, Seftel A, D'Souza D, Barry J: Efficacy of Continuous Dosing of Tadalafil Once Daily vs Tadalafil On Demand in Clinical Subgroups of Men With Erectile Dysfunction: A Descriptive Comparison Using the Integrated Tadalafil Databases. J Sex Med 2016, 13(5):860-875. 
16.Buvat J, Buttner H, Hatzimouratidis K, Vendeira PA, Moncada I, Boehmer M, Henneges C, Boess FG: Adherence to initial PDE-5 inhibitor treatment: randomized open-label study comparing tadalafil once a day, tadalafil on demand, and sildenafil on demand in patients with erectile dysfunction. J Sex Med 2013, 10(6):1592-1602.

17.Andersson KE: Mechanisms of penile erection and basis for pharmacological treatment of erectile dysfunction. Pharmacol Rev 2011, 63(4):811-859.

18.Francis SH, Busch JL, Corbin JD, Sibley D: cGMP-dependent protein kinases and cGMP phosphodiesterases in nitric oxide and cGMP action. Pharmacol Rev 2010, 62(3):525-563.

19.Liu Q, Zhang P, Pan J, Li Z, Liu J, Li G, Qin W, You Y, Yu X, Sun J et al: Cerebral Activity Changes in Different Traditional Chinese Medicine Patterns of Psychogenic Erectile Dysfunction Patients. Evid Based Complement Alternat Med 2015, 2015:503536.

20.Cera N, Delli Pizzi S, Di Pierro ED, Gambi F, Tartaro A, Vicentini C, Paradiso Galatioto G, Romani GL, Ferretti A: Macrostructural alterations of subcortical grey matter in psychogenic erectile dysfunction. PLoS One 2012, 7(6):e39118.

21.Zhang P, Liu J, Li G, Pan J, Li Z, Liu Q, Qin W, Dong M, Sun J, Huang X et al: White matter microstructural changes in psychogenic erectile dysfunction patients. Andrology 2014, 2(3):379-385.

22.Arnow BA, Desmond JE, Banner LL, Glover GH, Solomon A, Polan ML, Lue TF, Atlas SW: Brain activation and sexual arousal in healthy, heterosexual males. Brain 2002, 125(Pt 5):1014-1023.

23.Chen J, Chen Y, Chen G, Dai Y, Yao Z, Lu Q: Altered brain networks in psychogenic erectile dysfunction: a resting-state fMRI study. Andrology 2017, 5(6):1073-1081.

24.Chen J, Chen Y, Gao Q, Chen G, Dai Y, Yao Z, Lu Q: Brain structural network topological alterations of the left prefrontal and limbic cortex in psychogenic erectile dysfunction. Int J Neurosci 2018, 128(5):393403.

25.Chen J, Chen Y, Gao Q, Chen G, Dai Y, Yao Z, Lu Q: Impaired Prefrontal-Amygdala Pathway, SelfReported Emotion, and Erection in Psychogenic Erectile Dysfunction Patients With Normal Nocturnal Erection. Front Hum Neurosci 2018, 12:157.

26.Oh SK, Kim GW, Yang JC, Kim SK, Kang HK, Jeong GW: Brain activation in response to visually evoked sexual arousal in male-to-female transsexuals: 3.0 tesla functional magnetic resonance imaging. Korean J Radiol 2012, 13(3):257-264.

27.Georgiadis JR, Farrell MJ, Boessen R, Denton DA, Gavrilescu M, Kortekaas R, Renken RJ, Hoogduin JM, Egan GF: Dynamic subcortical blood flow during male sexual activity with ecological validity: a perfusion fMRI study. Neurolmage 2010, 50(1):208-216. 
28.Seo Y, Jeong B, Kim JW, Choi J: The relationship between age and brain response to visual erotic stimuli in healthy heterosexual males. Int J Impot Res 2010, 22(4):234-239.

29. Hayasaka S, Peiffer AM, Hugenschmidt CE, Laurienti PJ: Power and sample size calculation for neuroimaging studies by non-central random field theory. Neurolmage 2007, 37(3):721-730.

30.Desmond JE, Glover GH: Estimating sample size in functional MRI (fMRI) neuroimaging studies: statistical power analyses. J Neurosci Methods 2002, 118(2):115-128.

31.Chen L, Staubli SE, Schneider MP, Kessels AG, Ivic S, Bachmann LM, Kessler TM: Phosphodiesterase 5 inhibitors for the treatment of erectile dysfunction: a trade-off network meta-analysis. Eur Urol 2015, 68(4):674-680.

32.Deng $\mathrm{Y}$, Zhang $\mathrm{CH}$, Zhang $\mathrm{HN}$ : [Effects of chaihu shugan powder on the behavior and expressions of BDNF and TrkB in the hippocampus, amygdala, and the frontal lobe in rat model of depression]. Zhongguo Zhong xi yi jie he za zhi Zhongguo Zhongxiyi jiehe zazhi = Chinese journal of integrated traditional and Western medicine 2011, 31(10):1373-1378.

33.Kim SW, Sohn DW, Cho YH, Yang WS, Lee KU, Juh R, Ahn KJ, Chung YA, Han SI, Lee KH et al: Brain activation by visual erotic stimuli in healthy middle aged males. Int $J$ Impot Res 2006, 18(5):452-457.

34.Gao M, Yang X, Liu L, Fei N, Xi Y, Guo F, Yan F, Meng P, Yu L, Liu P et al: Abnormal White Matter Microstructure in Lifelong Premature Ejaculation Patients Identified by Tract-Based Spatial Statistical Analysis. J Sex Med 2018, 15(9):1272-1279.

35.Rifbjerg-Madsen S, Christensen AW, Boesen M, Christensen R, Danneskiold-Samsoe B, Bliddal H, Dreyer $\mathrm{L}$, Locht $\mathrm{H}$, Amris $\mathrm{K}$ : The course of pain hypersensitivity according to painDETECT in patients with rheumatoid arthritis initiating treatment: results from the prospective FRAME-cohort study. Arthritis Res Ther 2018, 20(1):105.

36.Yin S, Chen Y, Lei D, Sun RR, Ma TT, Feng PM, He ZX, Suo XL, Ma PH, Qu YZ et al: Cerebral mechanism of puncturing at He-Mu point combination for functional dyspepsia: study protocol for a randomized controlled parallel trial. Neural regeneration research 2017, 12(5):831-840.

37.Petzke F, Jensen KB, Kosek E, Choy E, Carville S, Fransson P, Williams SCR, Marcus H, Mainguy Y, Ingvar $\mathrm{M}$ et al: Using $\mathrm{fMRI}$ to evaluate the effects of milnacipran on central pain processing in patients with fibromyalgia. Scandinavian journal of pain 2013, 4(2):65-74.

38.Koopmann A, Bach P, Schuster R, Bumb JM, Vollstadt-Klein S, Reinhard I, Rietschel M, Witt SH, Wiedemann K, Kiefer F: Ghrelin modulates mesolimbic reactivity to alcohol cues in alcohol-addicted subjects: a functional imaging study. Addict Biol 2018.

39.Lu XW, Guo H, Sun JR, Dong QL, Zhao FT, Liao XH, Zhang L, Zhang Y, Li WH, Li ZX et al: A shared effect of paroxetine treatment on gray matter volume in depressive patients with and without childhood 
maltreatment: A voxel-based morphometry study. CNS neuroscience \& therapeutics 2018, 24(11):10731083.

40.Chan AW, Tetzlaff JM, Altman DG, Laupacis A, Gotzsche PC, Krleza-Jeric K, Hrobjartsson A, Mann H, Dickersin K, Berlin JA et al: SPIRIT 2013 statement: defining standard protocol items for clinical trials. Ann Intern Med 2013, 158(3):200-207.

41.Schulz KF, Altman DG, Moher D, Group C: CONSORT 2010 Statement: updated guidelines for reporting parallel group randomised trials. BMC Med 2010, 8:18.

42.Chan AW, Tetzlaff JM, Gotzsche PC, Altman DG, Mann H, Berlin JA, Dickersin K, Hrobjartsson A, Schulz KF, Parulekar WR et al: SPIRIT 2013 explanation and elaboration: guidance for protocols of clinical trials. BMJ 2013, 346:e7586.

43.Bai WJ, Li HJ, Jin JJ, Xu WP, Sebastian S, Wang XF: A randomized clinical trial investigating treatment choice in Chinese men receiving sildenafil citrate and tadalafil for treating erectile dysfunction. Asian $\mathrm{J}$ Androl 2017, 19(4):500-504.

44.Buvat J, Hatzichristou D, Boess FG, Buttner H, Gehchan N, Henneges C, Porst H: Continuation and effectiveness of tadalafil once daily during a 6-month observational study in erectile dysfunction: the EDATE study. Int J Clin Pract 2014, 68(9):1087-1099.

45.Rosen RC, Cappelleri JC, Gendrano N, 3rd: The International Index of Erectile Function (IIEF): a state-ofthe-science review. Int J Impot Res 2002, 14(4):226-244.

46. Rosen RC, Riley A, Wagner G, Osterloh IH, Kirkpatrick J, Mishra A: The international index of erectile function (IIEF): a multidimensional scale for assessment of erectile dysfunction. Urology 1997, 49(6):822830.

47.Lowy M, Collins S, Bloch M, Gillman M, Lording D, Sutherland P, Wang H, Stecher V: Quality of erection questionnaire correlates: change in erection quality with erectile function, hardness, and psychosocial measures in men treated with sildenafil for erectile dysfunction. J Sex Med 2007, 4(1):83-92.

48.Porst H, Gilbert C, Collins S, Huang X, Symonds T, Stecher V, Hvidsten K: Development and validation of the quality of erection questionnaire. J Sex Med 2007, 4(2):372-381.

49.Hvidsten K, Carlsson M, Stecher VJ, Symonds T, Levinson I: Clinically meaningful improvement on the quality of erection questionnaire in men with erectile dysfunction. Int $J$ Impot Res 2010, 22(1):45-50.

50.Mulhall JP, Goldstein I, Bushmakin AG, Cappelleri JC, Hvidsten K: Validation of the erection hardness score. J Sex Med 2007, 4(6):1626-1634.

51.Cappelleri JC, Althof SE, Siegel RL, Shpilsky A, Bell SS, Duttagupta S: Development and validation of the Self-Esteem And Relationship (SEAR) questionnaire in erectile dysfunction. Int J Impot Res 2004, 
16(1):30-38.

52. Hamilton M: The assessment of anxiety states by rating. The British journal of medical psychology 1959, 32(1):50-55.

53.Hamilton M: A rating scale for depression. J Neurol Neurosurg Psychiatry 1960, 23:56-62.

54.Berntsen EM, Rasmussen IA, Samuelsen P, Xu J, Haraldseth O, Lagopoulos J, Malhi GS: Putting the brain in Jeopardy: a novel comprehensive and expressive language task?Acta neuropsychiatrica 2006, 18(2):115-119.

55.Hao H, Chen C, Mao W, Zhong J, Dai Z: Aberrant brain regional homogeneity in first-episode drug-naive patients with major depressive disorder: A voxel-wise meta-analysis. Journal of affective disorders 2018, 245:63-71.

56.Smith SM, Jenkinson M, Johansen-Berg H, Rueckert D, Nichols TE, Mackay CE, Watkins KE, Ciccarelli O, Cader MZ, Matthews PM et al: Tract-based spatial statistics: voxelwise analysis of multi-subject diffusion data. Neurolmage 2006, 31(4):1487-1505.

57.Smith SM, Jenkinson M, Woolrich MW, Beckmann CF, Behrens TE, Johansen-Berg H, Bannister PR, De Luca M, Drobnjak I, Flitney DE et al: Advances in functional and structural MR image analysis and implementation as FSL. Neurolmage 2004, 23 Suppl 1:S208-219.

58.Zhao N, Yuan LX, Jia XZ, Zhou XF, Deng XP, He HJ, Zhong J, Wang J, Zang YF: Intra- and Inter-Scanner Reliability of Voxel-Wise Whole-Brain Analytic Metrics for Resting State fMRI. Frontiers in neuroinformatics 2018, 12:54.

59.Burnett AL, Nehra A, Breau RH, Culkin DJ, Faraday MM, Hakim LS, Heidelbaugh J, Khera M, McVary KT, Miner MM et al: Erectile Dysfunction: AUA Guideline. J Urol 2018.

60.Li L, Fan W, Li J, Li Q, Wang J, Fan Y, Ye T, Guo J, Li S, Zhang Y et al: Abnormal brain structure as a potential biomarker for venous erectile dysfunction: evidence from multimodal MRI and machine learning. Eur Radiol 2018, 28(9):3789-3800.

61.Wang Y, Dong M, Guan M, Wu J, He Z, Zou Z, Chen X, Shi D, Liang J, Zhang X: Aberrant Insula-Centered Functional Connectivity in Psychogenic Erectile Dysfunction Patients: A Resting-State fMRI Study. Front Hum Neurosci 2017, 11:221.

62.Zhao L, Guan M, Zhang X, Karama S, Khundrakpam B, Wang M, Dong M, Qin W, Tian J, Evans AC et al: Structural insights into aberrant cortical morphometry and network organization in psychogenic erectile dysfunction. Hum Brain Mapp 2015, 36(11):4469-4482.

63.Zhao L, Guan M, Zhu X, Karama S, Khundrakpam B, Wang M, Dong M, Qin W, Tian J, Evans AC et al: Aberrant Topological Patterns of Structural Cortical Networks in Psychogenic Erectile Dysfunction. Front 
Hum Neurosci 2015, 9:675.

\section{Tables}

Table 1: Time courses of serum bilibubin values 


\begin{tabular}{|c|c|c|c|c|c|c|}
\hline AEDs & doses $(\mathrm{mg} / \mathrm{d})$ & DOE & $\begin{array}{l}\text { TBil(0- } \\
25 \mu \mathrm{mol} / \mathrm{L})\end{array}$ & $\begin{array}{l}\text { DBil(0- } \\
7 \mu \mathrm{mol} / \mathrm{L})\end{array}$ & $\begin{array}{l}\text { IDBil(0- } \\
18 \mu \mathrm{mol} / \mathrm{L})\end{array}$ & \\
\hline DPK & 500 & \multirow{2}{*}{$\begin{array}{l}\text { Jun- } \\
2010\end{array}$} & 73.5 & 21.2 & 52.3 & \\
\hline LTG & 75 & & 71.4 & 20.0 & 51.4 & \\
\hline LTG & 50 & $\begin{array}{l}\text { Aug- } \\
2010\end{array}$ & 34.2 & 12.5 & 21.7 & \\
\hline LEV & 750 & \multirow{2}{*}{$\begin{array}{l}\text { Apr- } \\
2011\end{array}$} & 69.4 & 13.9 & 55.5 & \\
\hline LEV & 100 & & 41.1 & 12.7 & 28.4 & $* a$ \\
\hline LEV & 100 & $\begin{array}{l}\text { Jun- } \\
2011\end{array}$ & 136.8 & 9.5 & 127.3 & *b \\
\hline LEV & 100 & \multirow{2}{*}{$\begin{array}{l}\text { Jul- } \\
2011\end{array}$} & 23.4 & 8.2 & 15.2 & \multirow[t]{2}{*}{${ }^{*} \mathrm{C}$} \\
\hline LEV & 1000 & & 60.5 & 16.3 & 44.2 & \\
\hline LEV & 750 & $\begin{array}{l}\text { Jul- } \\
2011\end{array}$ & 23.6 & 6.6 & 17.0 & \multirow[t]{3}{*}{ Normal } \\
\hline LEV & 750 & \multirow{2}{*}{$\begin{array}{l}\text { Jul- } \\
2011\end{array}$} & 76.8 & 13.7 & 63.1 & \\
\hline LEV & 750 & & 43.3 & 11.6 & 31.7 & \\
\hline LEV & 750 & $\begin{array}{l}\text { Aug- } \\
2011\end{array}$ & 24.2 & 7.0 & 17.2 & \multirow[t]{4}{*}{ Normal } \\
\hline LEV & 750 & Jan- & 49.4 & 12.6 & 36.8 & \\
\hline LEV & 500 & \multirow{2}{*}{$\begin{array}{l}\text { Sep- } \\
2013\end{array}$} & 39.8 & 10.5 & 29.3 & \\
\hline LEV & 250 & & 45.9 & 12.7 & 33.2 & \\
\hline withdrawal & 0 & \multirow{2}{*}{$\begin{array}{l}\text { Mar- } \\
2014\end{array}$} & 50.9 & 17.5 & 33.4 & \multirow{3}{*}{ Normal } \\
\hline withdrawal & 0 & & 24.4 & 6.9 & 17.5 & \\
\hline \multirow[t]{7}{*}{ withdrawal } & 0 & \multirow{2}{*}{$\begin{array}{l}\text { Oct- } \\
2014 \\
\\
\text { Mar- } \\
2015\end{array}$} & 39.1 & 11.2 & 27.9 & \\
\hline & & & & & & \\
\hline & & $\begin{array}{l}\text { Mar- } \\
2016\end{array}$ & & & & \\
\hline & & $\begin{array}{l}\text { Apr- } \\
2017\end{array}$ & & & & \\
\hline & & $\begin{array}{l}\text { Nov- } \\
2017\end{array}$ & & & & \\
\hline & & $\begin{array}{l}\text { Mar- } \\
2018\end{array}$ & & & & \\
\hline & & $\begin{array}{l}\text { Aug- } \\
2018\end{array}$ & & & & \\
\hline
\end{tabular}


Table 10Time courses of serum bilibubin values when the patient was taking different AEDs under diverse daily doses. DOE: date of examination, TBil: total bilirubin, DBil: direct bilirubin, IDBil: indirect bilirubin;

*a: The bilirubin results before caloric restriction test. *b: The bilirubin results after the caloric restriction test. ${ }^{*} \mathrm{C}$ : The bilirubin results after oral phenytoin sodium stimulation test. And the reference values are as follows: TBIL $₫ 3.4 \sim 20.5 \mu \mathrm{mol} / \mathrm{L})$, DBIL 1.7 6.8 $\mu \mathrm{mol} / \mathrm{L}, \mathrm{IBIL} 6.8 \sim 12.0 \mu \mathrm{mol} / \mathrm{L}$.

\section{Figures}


Fig. 1 Flowchart of the study protocol

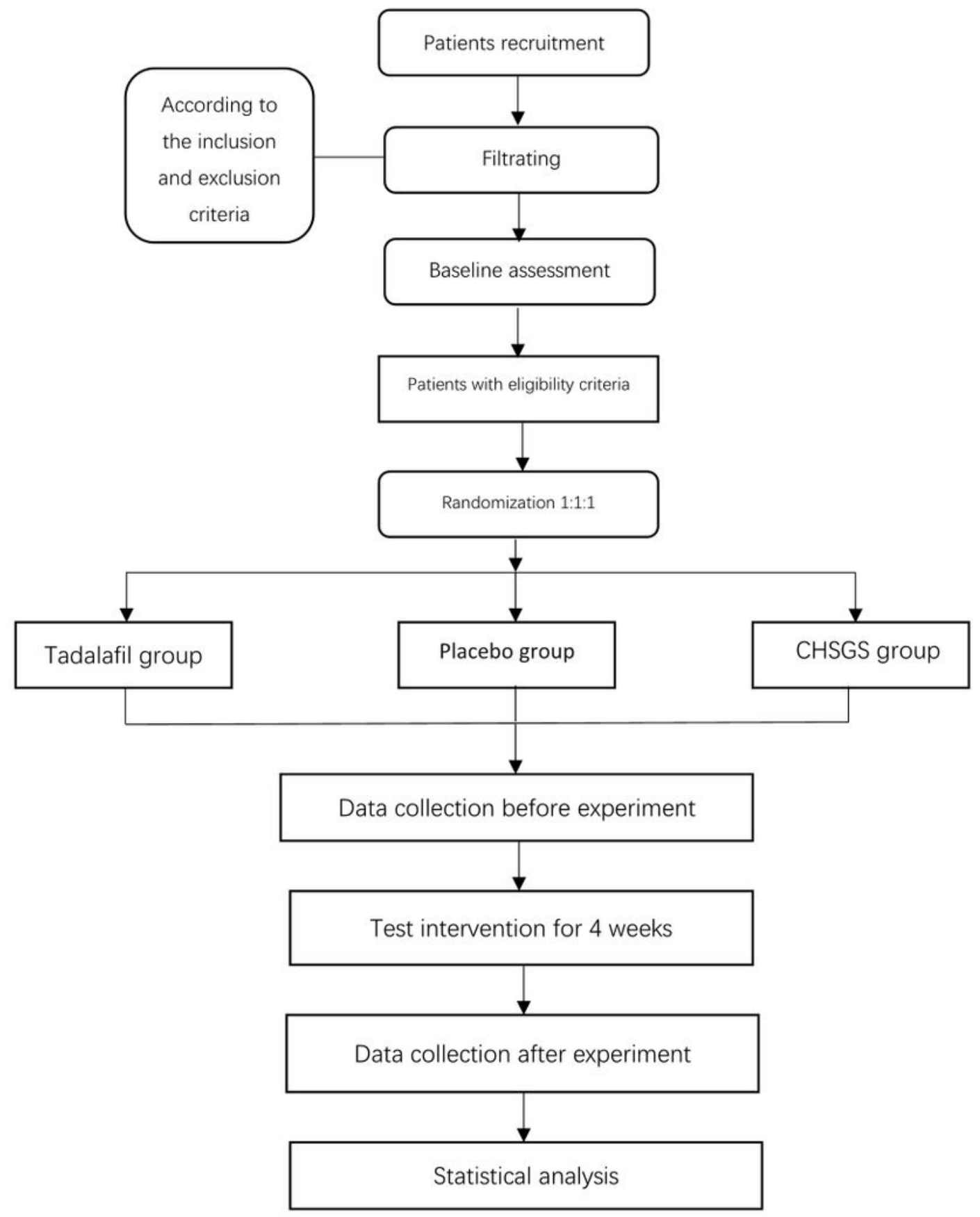

Figure 1

The Flowchart of the study protocol. 
Fig. 2 Standard Protocol Items

\begin{tabular}{|c|c|c|c|c|c|c|c|}
\hline & \multicolumn{7}{|c|}{ study protocol period } \\
\hline \multirow{2}{*}{ Time point } & \multirow[t]{2}{*}{ Enrolment } & \multirow[t]{2}{*}{ Distribution } & \multicolumn{5}{|c|}{$\begin{array}{l}\text { Post-distribution } \\
\text { (week) }\end{array}$} \\
\hline & & & 1 & 2 & 3 & 4 & 5 \\
\hline \multirow{5}{*}{$\begin{array}{l}\text { Enrolment: } \\
\text { Eligibility screen } \\
\text { Informed consent } \\
\text { Demographics } \\
\text { Allocation }\end{array}$} & & & & & & & \\
\hline & $x$ & & & & & & \\
\hline & $x$ & & & & & & \\
\hline & $x$ & & & & & & \\
\hline & & $x$ & & & & & \\
\hline \multicolumn{8}{|l|}{ Intervention: } \\
\hline \multicolumn{8}{|l|}{ Tadalafil group } \\
\hline \multicolumn{8}{|l|}{ Placebo group } \\
\hline \multicolumn{8}{|l|}{ Healthy subjects } \\
\hline \multicolumn{8}{|l|}{ Assessments: } \\
\hline \multirow{3}{*}{$\begin{array}{l}\text { HAMA-14 } \\
\text { HAMD-17 } \\
\text { Penile DUS }\end{array}$} & $x$ & & & & & $x$ & \\
\hline & $x$ & & & & & $x$ & \\
\hline & $x$ & & & & & $x$ & \\
\hline \multirow{2}{*}{$\begin{array}{l}\text { QEQ } \\
\text { IIEF-5 }\end{array}$} & $x$ & & & & & $x$ & \\
\hline & $x$ & & & & & $x$ & \\
\hline \multirow{2}{*}{$\begin{array}{l}\text { SEAR } \\
\text { NPT }\end{array}$} & $x$ & & & & & $x$ & \\
\hline & $x$ & & & & & $x$ & \\
\hline \multirow{2}{*}{$\begin{array}{l}\text { EHS } \\
\text { MRI }\end{array}$} & $x$ & & & & & $x$ & \\
\hline & $x$ & & & & & $x$ & \\
\hline Adverse events & & & $x$ & $x$ & $x$ & $x$ & Call-back \\
\hline
\end{tabular}

Abbreviation:

EHS: Erection hardness score

QEQ: Quality of erection questionnaire

NPT: Nocturnal penile tumescence

MRI: Magnetic resonance imaging

SEAR: Self-esteem and relationship questionnaire

IIEF-5: International index of erectile function 5

penile DUS: penile Duplex doppler ultrasonography

HAMA-14: 14-item Hamilton Rating Scale for Anxiety

HAMD-17: 17-item Hamilton Rating Scale for Depression

\section{Figure 2}

Standard Protocol Items. 
Fig 3 Analysis methods for outcomes

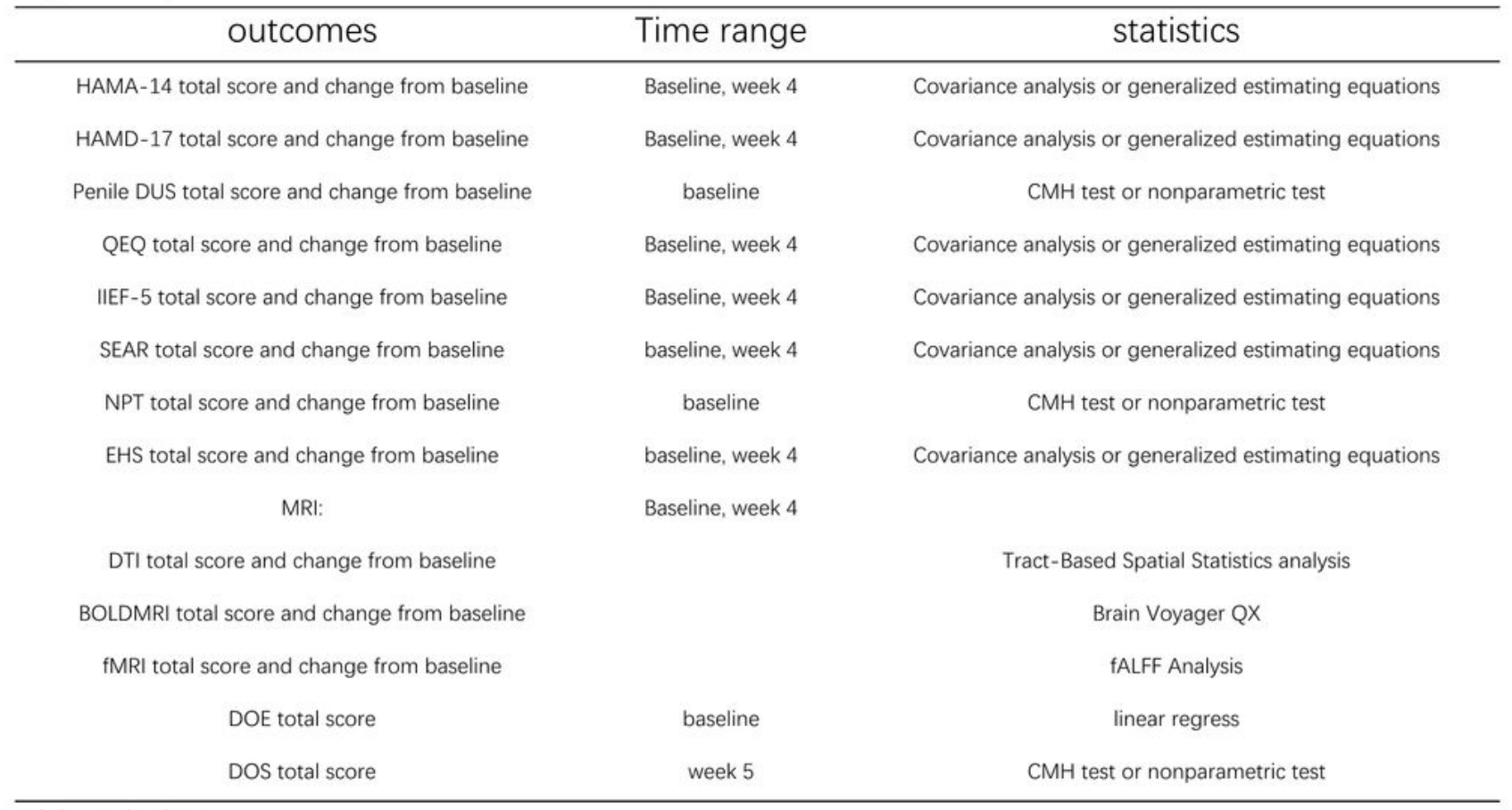

Abbreviation:

EHS: Erection hardness score

QEQ: Quality of erection questionnaire

NPT: Nocturnal penile tumescence

SEAR: Self-esteem and relationship questionnaire

IIEF-5: International index of erectile function 5

penile DUS: penile Duplex doppler ultrasonography

HAMA-14: 14-item Hamilton Rating Scale for Anxiety

HAMD-17: 17-item Hamilton Rating Scale for Depression

MRI: Magnetic resonance imaging

DTI: Diffusion tensor imaging

BOLDMRI: Blood oxygenation level dependent MRI

fMRI: functional MRI

DOE: Degree of expectation

DOS: Degree of satisfaction

CMH: Cochran-Mantel-Haenszel test

fALFF: fractional Amplitude of Low-Frequency Fluctuation Analysis

\section{Figure 3}

Analysis methods for outcomes. 
Fig 4 the central transmitters in brain regions

\begin{tabular}{|l|l|l|l|l|}
\hline Cognitive & Autonomic & Motivational & Emotional & Inhibitory \\
\hline Right lateral & ACC & PPC & SI & Medial OFC \\
\hline OFC & & SN & & LTC \\
\hline SPL & putamens & striatum & SIl & dmPFC \\
\hline SMA & & claustrum & & PCC \\
\hline ITC & hypothalamus & hypothalamus & pINS & left lateral \\
\hline IPL & & aMCC & & ANG \\
\hline PMv & alNS & & amygdala & OFC \\
\hline
\end{tabular}

abbreviation:

OFC, orbitofrontal cortex

ITC, inferior temporal cortex

IPL/SPL, inferior/superior parietal lobule

SMA, supplementary motor area

$\mathrm{PMv}$, ventral premotor area

ACC, anterior cingulate cortex

alNS/pINS, anterior/posterior insula

PPC, posterior parietal cortex (Brodmann area 40)

$\mathrm{SN}$, substantia nigra

aMCC, anterior middle cingulate cortex

$\mathrm{SI} / \mathrm{SII}$, primary/secondary somatosensory cortex

LTC, lateral temporal cortex

dmPFC, dorsal medial prefrontal cortex

PCC, posterior cingulate cortex

ANG, angular gyri

\section{Figure 4}

The central transmitters in brain regions.

\section{Supplementary Files}

This is a list of supplementary files associated with this preprint. Click to download. 
- supplement1.pdf

Page 25/25 